Brit. Heart f., 1968, 30, 60.

\title{
Haemodynamic Effects of Exercise in Isolated Pulmonary Stenosis Before and After Surgery
}

\author{
BENGT JONSSON AND SIMON J. K. LEE`
}

From the Department of Cardiology, Thoracic Clinic, Karolinska Sjukhuset, Stockholm, Sweden

Surgical treatment of pulmonary stenosis has frequently been evaluated by the reduction of pressure gradient across the valve. In only a few patients, however, have exercise tolerance and haemodynamic response to muscular work been reported both before and after operation. Johnson (1962) compared the findings in 7 patients after pulmonary valvotomy with those in 8 patients without operation. A decreased cardiac output during exercise was common to both groups. Because of differences between patients in such a group it seems preferable to study the same patient before and after operation.

Ikkos, Jonsson, and Linderholm (1966) reported the circulatory response to exercise in patients with pulmonary stenosis. Some of these patients have now been re-examined after operation and a comparison of their pre- and post-operative working capacity and haemodynamic findings constitutes this report.

\section{SuBJects AND METHODS}

All adult patients with pulmonary stenosis and intact ventricular septum who were investigated before operation at this laboratory and operated on between 1957 and 1964 have been readmitted to hospital 9 months or more after the operations, which were performed in the Department of Surgery, Thoracic Clinic, Karolinska Sjukhuset, either under hypothermia or with extracorporeal circulation. Patients operated on before 1957 were excluded from this study except for one (Case 17) who had an isolated infundibular pulmonary stenosis corrected in 1953. Of the remaining 16 patients, 12 had pulmonary valvotomy through the pulmonary artery, and 4 (Cases 3, 9, 13, and 15) had, in addition, resection of infundibular stenosis through a ventriculotomy incision.

The patients were divided into two groups. Group A (Cases 1-9) comprised those patients without an associ-

Received March 29, 1967.

* Travelling Fellow (Merck, Sharp and Dome) of the Royal College of Physicians of Canada. Present address: University of Alberta Hospital, Edmonton, Alberta, Canada. ated atrial septal defect; Group B (Cases 10-17) were those with either an atrial septal defect or a patent foramen ovale in addition to their pulmonary valvular lesion.

Such defects were not closed at operation. All patients were examined both at rest and during exercise at post-operative cardiac catheterization, though 5 patients were studied only at rest during their preoperative examination. However, an exercise tolerance test was carried out in all patients both before and after operation.

The methods used were those described in an earlier report (Ikkos et al., 1966). All blood gas analyses, except for 4 patients, were done with the spectrophotometric method which gave a normal arterial oxygen saturation of 98 per cent $(95-100 \%)$. Blood gases were measured by the Van Slyke method in Cases 14 to 17. When patients had arterial desaturation, the pulmonary flow was calculated by assuming that the pulmonary venous saturation was 98 per cent. In calculating the systemic flow in the presence of a left-to-right shunt at the atrial level, the blood in the superior vena cava was accepted as a representative sample of the mixed venous blood. In such patients systemic flow could not be calculated during exercise as it was not certain whether the left-to-right shunt existed during exercise. Those patients with a left-to-right shunt at rest who developed arterial desaturation during exercise are designated $(+)$ in Table IIB. When the shunt was only from right to left at rest it was assumed that no left-to-right shunt developed during exercise. The same assumption was also made if no shunt was found at rest and a right-toleft shunt appeared during exercise. All patients had normal lungs by radiological examination and spirometry.

Exercise tolerance tests were carried out according to the method previously described (Ikkos et al., 1966). All patients continued graded exercise to reach a heart rate of about 170 beats a minute and their physical working capacity was estimated at this level of exercise (PWC 170).

\section{Results}

Age, sex, body size, heart volume, physical working capacity, and the type of cardiac malformation 
TABLE I

BODY SIZE, HEART VOLUME, AND PHYSICAL WORKING CAPACITY BEFORE AND AFTER OPERATION

\begin{tabular}{|c|c|c|c|c|c|c|c|c|c|}
\hline $\begin{array}{l}\text { Case } \\
\text { No. }\end{array}$ & $\begin{array}{l}\text { Age at } \\
\text { opera- } \\
\text { tion } \\
(y r .)\end{array}$ & Sex & $\begin{array}{l}\text { Interval between } \\
\text { operation and } \\
\text { examination } \\
\text { (mth.) }\end{array}$ & $\begin{array}{l}\text { Height } \\
\text { (cm.) }\end{array}$ & $\begin{array}{l}\text { Weight } \\
\text { (kg.) }\end{array}$ & $\underset{\left(\mathbf{m} .^{2}\right)}{\text { B.S.A. }}$ & $\begin{array}{c}\text { Heart } \\
\text { vol. (ml.) }\end{array}$ & $\begin{array}{c}\text { Working } \\
\text { capacity } \\
(\mathrm{kpm} / \mathrm{min} .)\end{array}$ & $\begin{array}{l}\text { Type of heart } \\
\text { malformation }\end{array}$ \\
\hline 1 & 20 & $\mathbf{M}$ & $\begin{array}{l}\text { Pre-op. } \\
14 \text { Post-op. } \\
57 \text { Post-op. }\end{array}$ & $\begin{array}{l}170 \\
171 \\
172\end{array}$ & $\begin{array}{l}75 \\
71 \\
70\end{array}$ & $\begin{array}{l}1 \cdot 86 \\
1 \cdot 84 \\
1 \cdot 82\end{array}$ & $\begin{array}{l}800 \\
830 \\
770\end{array}$ & $\begin{array}{l}750 \\
825 \\
840\end{array}$ & Valve stenosis \\
\hline 2 & 27 & $\mathbf{F}$ & $\begin{array}{l}\text { Pre-op. } \\
10 \text { Post-op. }\end{array}$ & $\begin{array}{l}167 \\
168\end{array}$ & $\begin{array}{l}57 \\
58\end{array}$ & $\begin{array}{l}1.66 \\
1.67\end{array}$ & $\begin{array}{l}625 \\
710\end{array}$ & $\begin{array}{l}500 \\
420\end{array}$ & Valve stenosis \\
\hline 3 & 25 & $\mathbf{M}$ & $\begin{array}{l}\text { Pre-op. } \\
10 \text { Post-op. } \\
24 \text { Post-op. } \\
36 \text { Post-op. }\end{array}$ & $\begin{array}{l}172 \\
172 \\
172 \\
172\end{array}$ & $\begin{array}{l}64 \\
66 \\
66 \\
73\end{array}$ & $\begin{array}{l}1 \cdot 75 \\
1 \cdot 79 \\
1 \cdot 79 \\
\end{array}$ & $\begin{array}{c}1600 \\
1020 \\
\overline{760}\end{array}$ & $\begin{array}{l}450 \\
580 \\
580 \\
590\end{array}$ & $\begin{array}{l}\text { Valve and infundibular } \\
\text { stenosis }\end{array}$ \\
\hline 4 & 28 & $\mathbf{F}$ & $\begin{array}{l}\text { Pre-op. } \\
30 \text { Post-op. }\end{array}$ & $\begin{array}{l}159 \\
159\end{array}$ & $\begin{array}{l}59 \\
55\end{array}$ & $\begin{array}{l}1 \cdot 60 \\
1.56\end{array}$ & $\begin{array}{l}760 \\
860\end{array}$ & $\begin{array}{l}400 \\
570\end{array}$ & Valve stenosis \\
\hline 5 & 33 & $\mathbf{M}$ & $\begin{array}{l}\text { Pre-op. } \\
16 \text { Post-op. }\end{array}$ & $\begin{array}{l}172 \\
172\end{array}$ & $\begin{array}{l}75 \\
77\end{array}$ & $\begin{array}{l}1 \cdot 78 \\
1.90\end{array}$ & $\begin{array}{r}950 \\
1100\end{array}$ & $\begin{array}{l}900 \\
900\end{array}$ & Valve stenosis \\
\hline 6 & 39 & $\mathbf{F}$ & $\begin{array}{l}\text { Pre-op. } \\
10 \text { Post-op. } \\
41 \text { Post-op. }\end{array}$ & $\begin{array}{l}153 \\
154 \\
153\end{array}$ & $\begin{array}{l}56 \\
53 \\
54\end{array}$ & $\begin{array}{l}1.53 \\
1.50 \\
1.49\end{array}$ & $\begin{array}{r}1000 \\
920 \\
900\end{array}$ & $\begin{array}{l}625 \\
670 \\
600\end{array}$ & Valve stenosis \\
\hline 7 & 51 & $\mathbf{F}$ & $\begin{array}{l}\text { Pre-op. } \\
17 \text { Post-op. } \\
29 \text { Post-op. }\end{array}$ & $\begin{array}{l}165 \\
166 \\
165\end{array}$ & $\begin{array}{l}66 \\
64 \\
64\end{array}$ & $\begin{array}{l}1 \cdot 72 \\
1 \cdot 72 \\
1 \cdot 70\end{array}$ & $\begin{array}{r}1120 \\
880 \\
950\end{array}$ & $\begin{array}{l}450 \\
510 \\
500\end{array}$ & Valve stenosis \\
\hline 8 & 55 & $\mathbf{M}$ & $\begin{array}{l}\text { Pre-op. } \\
14 \text { Post-op. }\end{array}$ & $\begin{array}{l}163 \\
162\end{array}$ & $\begin{array}{l}76 \\
69\end{array}$ & $\begin{array}{l}1 \cdot 81 \\
1 \cdot 73\end{array}$ & $\begin{array}{r}800 \\
1000\end{array}$ & $\begin{array}{l}660 \\
690\end{array}$ & Valve stenosis \\
\hline 9 & 38 & $\mathbf{M}$ & Pre-op. & $\begin{array}{l}176 \\
176\end{array}$ & $\begin{array}{l}79 \\
78\end{array}$ & $\begin{array}{l}1.96 \\
1.94\end{array}$ & $\begin{array}{l}1220 \\
1150\end{array}$ & $\begin{array}{l}750 \\
900\end{array}$ & Valve stenosis \\
\hline 10 & 15 & $\mathbf{F}$ & $\begin{array}{l}\text { Pre-op. } \\
9 \\
36\end{array}$ & $\begin{array}{l}142 \\
144 \\
149\end{array}$ & $\begin{array}{l}38 \\
43 \\
51\end{array}$ & $\begin{array}{l}1 \cdot 22 \\
1 \cdot 29 \\
1 \cdot 42\end{array}$ & $\begin{array}{l}510 \\
510 \\
705\end{array}$ & $\begin{array}{l}250 \\
450 \\
550\end{array}$ & $\begin{array}{l}\text { Valve stenosis; intra- } \\
\text { atrial communication }\end{array}$ \\
\hline 11 & 19 & $\mathbf{M}$ & ${ }_{9}^{\text {Pre-op. }}$ & $\begin{array}{l}187 \\
192\end{array}$ & $\begin{array}{l}76 \\
86\end{array}$ & $\begin{array}{l}2 \cdot 04 \\
2 \cdot 18\end{array}$ & $\begin{array}{l}785 \\
880\end{array}$ & $\begin{array}{l}600 \\
660\end{array}$ & $\begin{array}{l}\text { Valve stenosis; intra- } \\
\text { atrial communication }\end{array}$ \\
\hline 13 & 22 & $\mathbf{F}$ & $\underset{9}{\text { Pre-op. }}$ & $\begin{array}{l}144 \\
143\end{array}$ & $\begin{array}{l}45 \\
41\end{array}$ & $\begin{array}{l}1 \cdot 32 \\
1 \cdot 26\end{array}$ & $\begin{array}{l}500 \\
581\end{array}$ & $\begin{array}{l}450 \\
400\end{array}$ & $\begin{array}{l}\text { Valve and infundibular } \\
\text { stenosis; intra-atrial } \\
\text { communication }\end{array}$ \\
\hline 14 & 24 & $\mathbf{M}$ & $\begin{array}{l}\text { Pre-op. } \\
91 \text { Post-op. }\end{array}$ & $\begin{array}{l}181 \\
181\end{array}$ & $\begin{array}{l}65 \\
71\end{array}$ & $\begin{array}{l}1.85 \\
1.95\end{array}$ & $\begin{array}{l}1220 \\
1240\end{array}$ & $\begin{array}{l}500 \\
840\end{array}$ & $\begin{array}{l}\text { Valve stenosis; intra- } \\
\text { atrial communication }\end{array}$ \\
\hline 15 & 31 & $\mathbf{F}$ & $\begin{array}{l}\text { Pre-op. } \\
30 \text { Post-op. }\end{array}$ & $\begin{array}{l}159 \\
159\end{array}$ & $\begin{array}{l}58 \\
56\end{array}$ & $\begin{array}{l}1 \cdot 60 \\
1 \cdot 58\end{array}$ & $\begin{array}{l}710 \\
915\end{array}$ & $\begin{array}{l}450 \\
550\end{array}$ & $\begin{array}{l}\text { Valve stenosis; intra- } \\
\text { atrial communication }\end{array}$ \\
\hline 16 & 36 & $\mathbf{M}$ & $\begin{array}{l}\text { Pre-op. } \\
15 \text { Post-op. }\end{array}$ & $\begin{array}{l}170 \\
170\end{array}$ & $\begin{array}{l}49 \\
52\end{array}$ & $\begin{array}{l}1 \cdot 57 \\
1 \cdot 61\end{array}$ & $\begin{array}{l}638 \\
680\end{array}$ & $\begin{array}{l}400 \\
450\end{array}$ & $\begin{array}{l}\text { Valve stenosis; intra- } \\
\text { atrial communication }\end{array}$ \\
\hline 17 & 29 & $M$ & $\begin{array}{l}\text { Pre-op. } \\
135 \text { Post-op. }\end{array}$ & $\begin{array}{l}174 \\
175\end{array}$ & $\begin{array}{l}65 \\
82\end{array}$ & $\begin{array}{l}1 \cdot 79 \\
1 \cdot 98\end{array}$ & $\begin{array}{l}860 \\
850\end{array}$ & $\begin{array}{l}600 \\
800\end{array}$ & $\begin{array}{l}\text { Infundibular stenosis; } \\
\text { intra-atrial } \\
\text { communication }\end{array}$ \\
\hline
\end{tabular}

are listed in Table $I$ and the findings at heart catheterization in Table II. At the final postoperative study the right ventricular systolic pressure at rest was between 52 and $71 \mathrm{~mm} . \mathrm{Hg}$ in 5 patients and below $40 \mathrm{~mm}$. $\mathrm{Hg}$ in the others. The estimated pulmonary valve area increased considerably after operation. The average valve area was 0.3 cm. ${ }^{2} / \mathrm{m}^{2}$ B.S.A. before operation and $1.2 \mathrm{~cm} .^{2} / \mathrm{m} .{ }^{2}$ B.S.A. after operation. Post-operatively, it was more than $1 \mathrm{~cm} .{ }^{2} / \mathrm{m}^{2}$ B.S.A. in 11 and between
0.49 and $0.97 \mathrm{~cm} .^{2} / \mathrm{m} .{ }^{2}$ B.S.A. in the remaining 6 patients.

The right ventricular end-diastolic pressure decreased significantly after operation in Group A, but the change was less in Group B (Fig. 1 and Table III). The rise in end-diastolic pressure during exercise was less conspicuous following operation. In all 17 patients studied after operation this pressure was measured during exercise and in only 4 was it more than $10 \mathrm{~mm}$. $\mathrm{Hg}$ (highest 
TABLE IIA

RESULTS OF HEART CATHETERIZATION IN 9 PATIENTS WITH INTACT ATRIAL SEPTUM

\begin{tabular}{|c|c|c|c|c|c|c|c|c|c|c|c|c|c|c|c|c|c|c|c|}
\hline \multirow{3}{*}{ 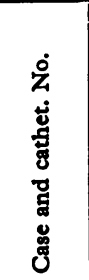 } & \multirow{3}{*}{ 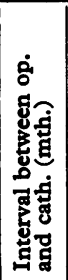 } & \multirow{3}{*}{ 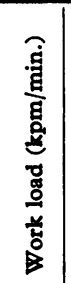 } & \multirow{3}{*}{ 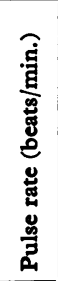 } & \multirow{3}{*}{ 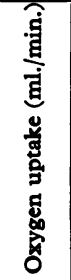 } & \multirow{3}{*}{ 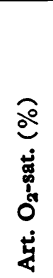 } & \multirow{3}{*}{ 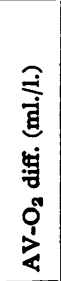 } & \multirow{3}{*}{ 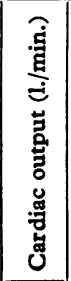 } & \multirow{3}{*}{ 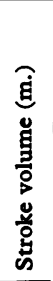 } & \multicolumn{9}{|c|}{ Pressures (mm. Hg) } & \multirow{3}{*}{ 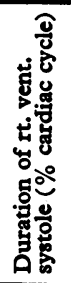 } & \multirow{3}{*}{ 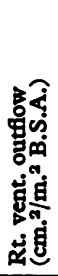 } \\
\hline & & & & & & & & & \multirow{2}{*}{$\begin{array}{c}\begin{array}{c}\text { Rt. } \\
\text { atr. }\end{array} \\
\text { Mean }\end{array}$} & \multicolumn{2}{|c|}{$\begin{array}{c}\text { Rt. } \\
\text { ventr. }\end{array}$} & \multicolumn{3}{|c|}{ Pulm. artery } & \multicolumn{3}{|c|}{ Aorta } & & \\
\hline & & & & & & & & & & Syst. & $\begin{array}{l}\text { End- } \\
\text { diast. }\end{array}$ & Syst. & Diast. & Mean & Syst. & Diast. & Mean & & \\
\hline $\begin{array}{c}1 \\
62 / 59 \\
147 / 61 \\
75 / 65\end{array}$ & $\begin{array}{l}14 \\
57\end{array}$ & $\begin{array}{c}\text { Rest } \\
250 \\
500 \\
\text { Rest } \\
700 \\
\text { Rest } \\
400 \\
800\end{array}$ & $\begin{array}{r}70 \\
118 \\
152 \\
64 \\
151 \\
62 \\
100 \\
163\end{array}$ & $\begin{array}{r}277 \\
928 \\
1362 \\
262 \\
1726 \\
248 \\
1119 \\
1988\end{array}$ & $\begin{array}{r}99 \\
98 \\
97 \\
97 \\
96 \\
100 \\
98 \\
97\end{array}$ & $\begin{array}{r}46 \\
92 \\
110 \\
46 \\
117 \\
46 \\
104 \\
119\end{array}$ & \begin{tabular}{|r|}
$6 \cdot 0$ \\
$10 \cdot 1$ \\
$12 \cdot 4$ \\
$56 \cdot 7$ \\
14.7 \\
5.4 \\
$10 \cdot 8$ \\
16.7 \\
\end{tabular} & $\begin{array}{r}86 \\
85 \\
81 \\
89 \\
98 \\
87 \\
108 \\
103\end{array}$ & $\begin{array}{l}- \\
7 \\
7 \\
4 \\
-\end{array}$ & $\begin{array}{l}111 \\
-49 \\
73 \\
37 \\
42 \\
76\end{array}$ & $\begin{array}{r}9 \\
-8 \\
3 \\
5 \\
3 \\
10\end{array}$ & $\begin{array}{l}15 \\
16 \\
19 \\
14 \\
31 \\
39\end{array}$ & $\begin{array}{r}5 \\
-6 \\
7 \\
5 \\
9 \\
7\end{array}$ & $\begin{array}{r}- \\
\overline{14} \\
14 \\
9 \\
18 \\
19\end{array}$ & $\begin{array}{l}145 \\
167 \\
119 \\
185 \\
111 \\
144 \\
174\end{array}$ & $\begin{array}{l}73 \\
70 \\
67 \\
79 \\
67 \\
76 \\
87\end{array}$ & $\begin{array}{r}89 \\
116 \\
87 \\
104 \\
121\end{array}$ & $\begin{array}{l}51 \\
39 \\
39\end{array}$ & $\begin{array}{l}0.33 \\
0.81 \\
1.02\end{array}$ \\
\hline $\begin{array}{r}22 \\
31 / 62 \\
9 / 63\end{array}$ & $\begin{array}{c}\text { Pre } \\
10\end{array}$ & $\begin{array}{l}\text { Rest } \\
300 \\
\text { Rest } \\
300\end{array}$ & $\begin{array}{r}64 \\
130 \\
71 \\
138\end{array}$ & $\begin{array}{l}282 \\
963 \\
229 \\
865\end{array}$ & $\begin{array}{l}99 \\
96 \\
98 \\
99\end{array}$ & $\begin{array}{l}40 \\
82 \\
33 \\
75\end{array}$ & \begin{tabular}{r|}
$7 \cdot 0$ \\
$11 \cdot 7$ \\
7.0 \\
11.6
\end{tabular} & $\begin{array}{r}109 \\
90 \\
98 \\
84\end{array}$ & $\begin{array}{r}11 \\
10 \\
6 \\
7\end{array}$ & $\begin{array}{r}84 \\
131 \\
32 \\
67\end{array}$ & $\begin{array}{r}10 \\
17 \\
5 \\
3\end{array}$ & $\frac{18}{16}$ & $\begin{array}{l}8 \\
5 \\
5\end{array}$ & $\begin{array}{l}11 \\
10 \\
13 \\
12\end{array}$ & $\begin{array}{l}132 \\
156 \\
124 \\
160\end{array}$ & $\begin{array}{l}77 \\
68 \\
74 \\
83\end{array}$ & $\begin{array}{r}- \\
98 \\
110\end{array}$ & $\begin{array}{l}50 \\
48\end{array}$ & $\begin{array}{l}0.53 \\
1.24\end{array}$ \\
\hline $\begin{array}{c}3 \\
171 / 58 \\
120 / 59\end{array}$ & $\begin{array}{c}\text { Pre } \\
10\end{array}$ & $\begin{array}{c}\text { Rest } \\
\text { Rest } \\
250 \\
500 \\
\text { Rest } \\
\text { Rest } \\
400\end{array}$ & $\begin{array}{r}74 \\
74 \\
119 \\
169 \\
71 \\
75 \\
120\end{array}$ & $\begin{array}{r}250 \\
255 \\
841 \\
1257 \\
245 \\
251 \\
1089\end{array}$ & $\begin{array}{r}99 \\
96 \\
99 \\
99 \\
95 \\
98 \\
100\end{array}$ & $\begin{array}{r}93 \\
52 \\
104 \\
139 \\
38 \\
46 \\
110\end{array}$ & $\begin{array}{l}2 \cdot 7 \\
4 \cdot 9 \\
8 \cdot 1 \\
9 \cdot 1 \\
6.4 \\
5 \cdot 4 \\
9 \cdot 9\end{array}$ & $\begin{array}{l}37 \\
67 \\
69 \\
54 \\
90 \\
72 \\
83\end{array}$ & $\begin{array}{l}\frac{12}{10} \\
\frac{9}{-}\end{array}$ & $\begin{array}{r}167 \\
63 \\
130 \\
164 \\
56 \\
52 \\
-\end{array}$ & $\begin{array}{r}19 \\
9 \\
6 \\
5 \\
6 \\
10 \\
-\end{array}$ & $\begin{array}{l}\overline{25} \\
31 \\
45 \\
20 \\
25 \\
30\end{array}$ & $\begin{array}{r}\overline{12} \\
13 \\
7 \\
7 \\
11 \\
18\end{array}$ & $\begin{array}{l}13 \\
19 \\
24 \\
24 \\
18 \\
17 \\
24\end{array}$ & $\begin{array}{l}116 \\
105 \\
158 \\
187 \\
128 \\
185\end{array}$ & $\begin{array}{l}74 \\
68 \\
81 \\
87 \\
78 \\
91\end{array}$ & $\begin{array}{r}87 \\
83 \\
103 \\
113 \\
97 \\
124\end{array}$ & $\begin{array}{l}55 \\
52\end{array}$ & $\begin{array}{l}0.13 \\
0.40 \\
\\
0.60 \\
0.69\end{array}$ \\
\hline $\begin{array}{c}4 \\
69 / 61 \\
205 / 64\end{array}$ & Pre & $\begin{array}{l}\text { Rest } \\
150 \\
300 \\
\text { Rest } \\
250 \\
500\end{array}$ & $\begin{array}{r}64 \\
108 \\
154 \\
62 \\
115 \\
168\end{array}$ & $\begin{array}{r}181 \\
502 \\
684 \\
195 \\
803 \\
1277\end{array}$ & $\begin{array}{r}99 \\
98 \\
98 \\
100 \\
99 \\
98\end{array}$ & $\begin{array}{r}54 \\
97 \\
119 \\
61 \\
115 \\
135\end{array}$ & $\begin{array}{l}3.3 \\
5.2 \\
5.6 \\
3.22 \\
6.98 \\
9.45\end{array}$ & $\begin{array}{l}52 \\
45 \\
37 \\
52 \\
61 \\
56\end{array}$ & $\frac{13}{\frac{13}{2}}$ & $\begin{array}{r}142 \\
178 \\
185 \\
23 \\
36 \\
40\end{array}$ & $\begin{array}{r}14 \\
15 \\
20 \\
4 \\
6 \\
3\end{array}$ & $\begin{array}{l}22 \\
26 \\
26 \\
17 \\
25 \\
26\end{array}$ & $\begin{array}{l}9 \\
7 \\
9 \\
7 \\
7 \\
9\end{array}$ & $\begin{array}{l}\bar{Z} \\
\overline{12} \\
13 \\
16\end{array}$ & $\begin{array}{l}122 \\
128 \\
122 \\
122 \\
137 \\
134\end{array}$ & $\begin{array}{l}77 \\
77 \\
82 \\
83 \\
76 \\
88\end{array}$ & $\begin{array}{r}96 \\
94 \\
100 \\
105 \\
107 \\
111\end{array}$ & 42 & $\begin{array}{r}0.20 \\
1.27\end{array}$ \\
\hline $\begin{array}{c}5 \\
145 / 61 \\
10 / 63\end{array}$ & Pre & $\begin{array}{l}\text { Rest } \\
400 \\
800 \\
\text { Rest } \\
400 \\
800\end{array}$ & $\begin{array}{r}85 \\
111 \\
149 \\
69 \\
97 \\
135\end{array}$ & $\begin{array}{r}350 \\
1141 \\
1835 \\
283 \\
1016 \\
1680\end{array}$ & $\begin{array}{l}98 \\
97 \\
98 \\
96 \\
98 \\
95\end{array}$ & $\begin{array}{r}50 \\
94 \\
115 \\
49 \\
98 \\
112\end{array}$ & \begin{tabular}{r|}
$7 \cdot 0$ \\
$12 \cdot 2$ \\
$16 \cdot 0$ \\
$5 \cdot 8$ \\
$10 \cdot 4$ \\
$15 \cdot 0$
\end{tabular} & $\begin{array}{r}82 \\
110 \\
107 \\
84 \\
107 \\
111\end{array}$ & $\frac{5}{6}$ & $\begin{array}{r}82 \\
112 \\
159 \\
35 \\
61 \\
81\end{array}$ & $\begin{array}{r}6 \\
12 \\
9 \\
6 \\
11 \\
5\end{array}$ & $\begin{array}{l}19 \\
27 \\
26 \\
18 \\
30 \\
32\end{array}$ & $\begin{array}{r}7 \\
8 \\
2 \\
9 \\
12 \\
13\end{array}$ & $\begin{array}{l}14 \\
16 \\
18 \\
13 \\
21 \\
18\end{array}$ & $\begin{array}{l}119 \\
147 \\
186 \\
126 \\
154 \\
171\end{array}$ & $\begin{array}{l}74 \\
78 \\
81 \\
79 \\
83 \\
80\end{array}$ & $\begin{array}{r}91 \\
105 \\
116 \\
98 \\
108\end{array}$ & 46 & $\begin{array}{l}0.63 \\
0.97\end{array}$ \\
\hline $\begin{array}{c}6 \\
124 / 62 \\
28 / 65\end{array}$ & $\begin{array}{c}\text { Pre } \\
10\end{array}$ & $\begin{array}{l}\text { Rest } \\
\text { Rest } \\
400 \\
\text { Rest } \\
300\end{array}$ & $\begin{array}{r}60 \\
69 \\
130 \\
79 \\
110\end{array}$ & $\begin{array}{r}254 \\
240 \\
1065 \\
278 \\
890\end{array}$ & $\begin{array}{l}95 \\
99 \\
97 \\
99 \\
99\end{array}$ & $\begin{array}{r}46 \\
42 \\
103 \\
43 \\
98\end{array}$ & $\begin{array}{r}5 \cdot 6 \\
5.7 \\
10.4 \\
6.5 \\
9 \cdot 1\end{array}$ & $\begin{array}{l}58 \\
83 \\
80 \\
82 \\
82\end{array}$ & $\begin{array}{l}7 \\
\frac{7}{2} \\
2 \\
9\end{array}$ & $\begin{array}{r}125 \\
60 \\
90 \\
29 \\
62\end{array}$ & $\begin{array}{r}8 \\
6 \\
17 \\
2 \\
12\end{array}$ & $\begin{array}{r}20 \\
21 \\
40 \\
9 \\
26\end{array}$ & $\begin{array}{r}11 \\
7 \\
12 \\
3 \\
12\end{array}$ & $\begin{array}{r}14 \\
13 \\
24 \\
5 \\
18\end{array}$ & $\begin{array}{l}\overline{137} \\
162 \\
136 \\
171\end{array}$ & $\begin{array}{l}\overline{64} \\
71 \\
72 \\
84\end{array}$ & $\begin{array}{r}- \\
86 \\
114 \\
95 \\
129\end{array}$ & $\begin{array}{l}54 \\
48 \\
46\end{array}$ & $\begin{array}{l}0.33 \\
0.80 \\
1.13\end{array}$ \\
\hline $\begin{array}{c}7 \\
4 / 62 \\
68 / 64 \\
72 / 65\end{array}$ & $\begin{array}{r}\text { Pre } \\
17\end{array}$ & $\begin{array}{l}\text { Rest } \\
350 \\
\text { Rest } \\
300 \\
450 \\
\text { Rest }\end{array}$ & $\begin{array}{r}60 \\
139 \\
125 \\
144 \\
58\end{array}$ & $\begin{array}{r}255 \\
1044 \\
\overline{874} \\
1112 \\
261\end{array}$ & $\begin{array}{r}97 \\
100 \\
98 \\
100 \\
100\end{array}$ & $\begin{array}{r}60 \\
131 \\
118 \\
134 \\
53\end{array}$ & $\begin{array}{l}4 \cdot 2 \\
8 \cdot 0 \\
7 \cdot 1 \\
8 \cdot 3 \\
4 \cdot 9\end{array}$ & $\begin{array}{l}70 \\
57 \\
59 \\
58 \\
83\end{array}$ & $\frac{\frac{12}{4}}{\frac{9}{9}}$ & $\begin{array}{r}156 \\
76 \\
133 \\
141 \\
62\end{array}$ & $\begin{array}{r}11 \\
3 \\
8 \\
7 \\
7\end{array}$ & $\begin{array}{l}20 \\
29 \\
16 \\
28 \\
28 \\
24\end{array}$ & $\begin{array}{r}11 \\
8 \\
6 \\
14 \\
13 \\
4\end{array}$ & $\begin{array}{l}10 \\
20 \\
12 \\
19 \\
19 \\
10\end{array}$ & $\begin{array}{l}134 \\
163 \\
144 \\
179 \\
180 \\
146\end{array}$ & $\begin{array}{l}72 \\
82 \\
79 \\
89 \\
93 \\
85\end{array}$ & $\begin{array}{r}96 \\
115 \\
105 \\
131 \\
124 \\
112\end{array}$ & $\begin{array}{l}56 \\
51 \\
43\end{array}$ & 0.22 \\
\hline $\begin{array}{c}8 \\
72 / 64 \\
119 / 65\end{array}$ & Pre & $\begin{array}{l}\text { Rest } \\
600 \\
\text { Rest } \\
600\end{array}$ & $\begin{array}{r}74 \\
148 \\
73 \\
143\end{array}$ & $\begin{array}{r}276 \\
1288 \\
288 \\
1525\end{array}$ & $\begin{array}{l}99 \\
97 \\
96 \\
98\end{array}$ & $\begin{array}{r}58 \\
121 \\
55 \\
138\end{array}$ & $\begin{array}{r}4 \cdot 8 \\
10 \cdot 7 \\
5.2 \\
11 \cdot 0\end{array}$ & $\begin{array}{l}64 \\
72 \\
71 \\
77\end{array}$ & 三 & $\begin{array}{r}70 \\
120 \\
29 \\
50\end{array}$ & $\begin{array}{r}3 \\
18 \\
2 \\
12\end{array}$ & $\begin{array}{l}23 \\
25 \\
24 \\
48\end{array}$ & $\begin{array}{r}8 \\
18 \\
8 \\
27\end{array}$ & $\begin{array}{l}17 \\
22 \\
14 \\
35\end{array}$ & $\begin{array}{l}150 \\
205 \\
138 \\
223\end{array}$ & $\begin{array}{r}84 \\
103 \\
81 \\
121\end{array}$ & $\begin{array}{l}117 \\
140 \\
105 \\
167\end{array}$ & 47 & $\begin{array}{l}0.40 \\
1.71\end{array}$ \\
\hline $\begin{array}{r}9 \\
122 / 59 \\
83 / 65\end{array}$ & $\begin{array}{l}\text { Pre } \\
5 \text { yr. }\end{array}$ & $\begin{array}{l}\text { Rest } \\
\text { Rest } \\
400 \\
800\end{array}$ & $\begin{array}{r}70 \\
83 \\
124 \\
160\end{array}$ & $\begin{array}{r}311 \\
383 \\
1347 \\
2311\end{array}$ & $\begin{array}{r}97 \\
100 \\
96 \\
95\end{array}$ & $\begin{array}{r}55 \\
53 \\
117 \\
149\end{array}$ & $\begin{array}{r}5.7 \\
7.3 \\
11.5 \\
15.5\end{array}$ & $\begin{array}{l}81 \\
87 \\
93 \\
97\end{array}$ & $\begin{array}{l}7 \\
\frac{4}{-}\end{array}$ & $\begin{array}{r}134 \\
\frac{38}{98} \\
98\end{array}$ & $\begin{array}{r}15 \\
\frac{6}{11}\end{array}$ & $\begin{array}{l}17 \\
22 \\
29 \\
44\end{array}$ & $\begin{array}{r}10 \\
3 \\
8 \\
6\end{array}$ & $\begin{array}{l}\overline{12} \\
17 \\
20\end{array}$ & $\begin{array}{l}128 \\
112 \\
162 \\
164\end{array}$ & $\begin{array}{l}69 \\
67 \\
84 \\
83\end{array}$ & $\begin{array}{r}86 \\
85 \\
113 \\
112\end{array}$ & $\begin{array}{l}54 \\
51\end{array}$ & $\begin{array}{l}0.30 \\
0.79\end{array}$ \\
\hline
\end{tabular}

The reference level for zero pressure was taken as $5 \mathrm{~cm}$. below the insertion of the fourth rib at the sternum with the patient in recumbent position. 
TABLE IIB

RESULTS OF HEART CATHETERIZATION IN 8 PATIENTS WITH INTRA-ATRIAL COMMUNICATION

\begin{tabular}{|c|c|c|c|c|c|c|c|c|c|c|c|c|c|c|c|c|c|c|c|c|c|c|c|c|}
\hline \multirow{3}{*}{ 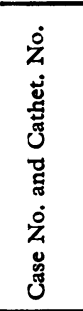 } & \multirow{3}{*}{ 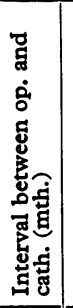 } & \multirow{3}{*}{ 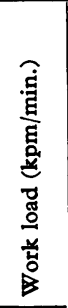 } & \multirow{3}{*}{ 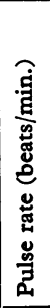 } & \multirow{3}{*}{ 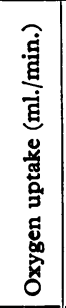 } & \multirow{3}{*}{ 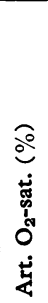 } & \multicolumn{2}{|c|}{$\begin{array}{c}\mathrm{AV}-\mathrm{O}_{2} \\
\text { diff. } \\
\text { (ml./1.) }\end{array}$} & \multicolumn{2}{|c|}{$\begin{array}{c}\text { Cardiac } \\
\text { output } \\
\text { (1./min.) }\end{array}$} & \multicolumn{2}{|c|}{$\begin{array}{c}\text { Stroke } \\
\text { vol. } \\
\text { (ml.) }\end{array}$} & \multirow{3}{*}{ 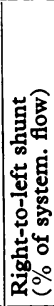 } & \multirow{3}{*}{ 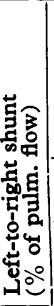 } & \multicolumn{9}{|c|}{ Pressures (mm. Hg) } & \multirow{3}{*}{ 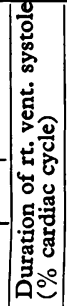 } & \multirow{3}{*}{ 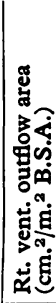 } \\
\hline & & & & & & 这 & . & . & ن. & . & ن. & & & \multirow{2}{*}{ 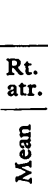 } & & & & & & & Aorts & & & \\
\hline & & & & & & 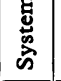 & 刍 & 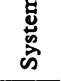 & 皇 & 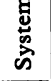 & 哥 & & & & 放 & 苟苛 & & 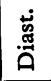 & & 岕 & $\begin{array}{l}\dot{\Delta} \\
\dot{\Delta}\end{array}$ & 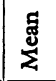 & & \\
\hline $\begin{array}{c}10 \\
84 / 60 \\
38 / 61\end{array}$ & $\begin{array}{r}\text { Pre } \\
9 \\
36\end{array}$ & & $\begin{array}{r}98 \\
84 \\
145 \\
82 \\
136\end{array}$ & $\begin{array}{r}245 \\
185 \\
766 \\
268 \\
1110\end{array}$ & $\begin{array}{l}8 \\
9 \\
7 \\
9 \\
8\end{array}$ & $\begin{array}{c}28 \\
\frac{42}{33} \\
-\end{array}$ & $\begin{array}{l}65 \\
34 \\
81 \\
23 \\
75\end{array}$ & $\begin{array}{l}8 \cdot 9 \\
4 \cdot 4 \\
\frac{-}{8 \cdot 2} \\
-\end{array}$ & $\begin{array}{r}3.8 \\
5.4 \\
9.5 \\
11.7 \\
14.8\end{array}$ & $\begin{array}{c}91 \\
53 \\
\frac{100}{-}\end{array}$ & $\begin{array}{r}38 \\
64 \\
66 \\
142 \\
109\end{array}$ & $\begin{array}{r}57 \\
20 \\
+ \\
0 \\
+\end{array}$ & $\begin{array}{r}0 \\
35 \\
30 \\
\end{array}$ & $\begin{array}{l}4 \\
6 \\
5 \\
8\end{array}$ & $\begin{array}{l}6 \\
60 \\
0 \\
9\end{array}$ & $\begin{array}{l}2 \\
2 \\
4 \\
4 \\
1\end{array}$ & $\frac{27}{25}$ & $\begin{array}{r}4 \\
8 \\
5 \\
14\end{array}$ & $\frac{8}{13}$ & $\begin{array}{r}83 \\
115 \\
147 \\
118 \\
126\end{array}$ & $\begin{array}{l}5 \\
5 \\
9 \\
7 \\
2\end{array}$ & $\begin{array}{l}97 \\
94\end{array}$ & 50 & $3 \cdot 2$ \\
\hline $\begin{array}{c}11 \\
34 / 63\end{array}$ & Pre & $\begin{array}{l}\text { Rest } \\
1000 \\
\text { Rest } \\
400 \\
800\end{array}$ & $\begin{array}{r}100 \\
196 \\
82 \\
133 \\
180\end{array}$ & $\begin{array}{r}302 \\
2166 \\
329 \\
1295 \\
2098\end{array}$ & $\begin{array}{l}98 \\
88 \\
99 \\
98 \\
98\end{array}$ & \begin{tabular}{|r|}
37 \\
118 \\
33 \\
86 \\
108 \\
\end{tabular} & \begin{tabular}{|r|}
37 \\
140 \\
33 \\
86 \\
108 \\
\end{tabular} & $\begin{array}{r}8 \cdot 2 \\
18 \cdot 4 \\
10 \cdot 0 \\
15 \cdot 0 \\
19.5\end{array}$ & $\begin{array}{r}8.2 \\
15.6 \\
10.0 \\
15.0 \\
19.5\end{array}$ & $\begin{array}{r}82 \\
94 \\
122 \\
113\end{array}$ & $\begin{array}{r}82 \\
80 \\
122 \\
113 \\
108\end{array}$ & $\begin{array}{r}0 \\
15 \\
0 \\
0 \\
0\end{array}$ & $\begin{array}{l}0 \\
0 \\
0 \\
0 \\
0\end{array}$ & $\frac{\overline{4}}{\overline{4}}$ & $\begin{array}{r}96 \\
176 \\
33 \\
78 \\
95\end{array}$ & $\begin{array}{r}3 \\
14 \\
4 \\
4 \\
9\end{array}$ & & $\begin{array}{l}1 \\
8 \\
7 \\
6 \\
8\end{array}$ & $\begin{array}{l}4 \\
21 \\
10 \\
18\end{array}$ & $\begin{array}{l}133 \\
210 \\
142 \\
172 \\
190\end{array}$ & & $\frac{-}{101}$ & 60 & .41 \\
\hline $95 / 63$ & $\begin{array}{l}\text { Pre } \\
36\end{array}$ & $\begin{array}{l}\text { Rest } \\
300 \\
\text { Rest } \\
600\end{array}$ & $\begin{array}{r}68 \\
121 \\
65 \\
108\end{array}$ & $\begin{array}{r}279 \\
948 \\
419 \\
1726 \\
\end{array}$ & $\begin{array}{l}91 \\
80 \\
98 \\
96\end{array}$ & $\begin{array}{r}73 \\
137 \\
44 \\
-\end{array}$ & $\begin{array}{r}92 \\
182 \\
33 \\
81\end{array}$ & $\begin{array}{l}3 \cdot 8 \\
6 \cdot 9 \\
9 \cdot 6 \\
-\end{array}$ & $\begin{array}{r}3.0 \\
5.2 \\
12.6 \\
21.3\end{array}$ & $\begin{array}{r}56 \\
57 \\
147\end{array}$ & $\begin{array}{r}45 \\
43 \\
195 \\
197\end{array}$ & $\begin{array}{r}21 \\
25 \\
0 \\
0\end{array}$ & $\begin{array}{r}0 \\
0 \\
24 \\
-\end{array}$ & $\begin{array}{r}8 \\
17 \\
9 \\
\end{array}$ & & $\begin{array}{r}14 \\
35 \\
7 \\
10\end{array}$ & $\frac{10}{17}$ & $\frac{0}{5}$ & $\overline{7}$ & $\begin{array}{l}114 \\
159 \\
120 \\
169\end{array}$ & & \begin{tabular}{|l|}
84 \\
91
\end{tabular} & 55 & $\cdot 16$ \\
\hline $\begin{array}{c}13 \\
91 / 61 \\
67 / 62\end{array}$ & $\begin{array}{r}\text { Pre } \\
9\end{array}$ & $\begin{array}{l}\text { Rest } \\
\text { Rest } \\
300\end{array}$ & $\begin{array}{r}88 \\
82 \\
131\end{array}$ & $\begin{array}{l}202 \\
251 \\
723\end{array}$ & & $\begin{array}{l}48 \\
40 \\
74\end{array}$ & $\begin{array}{l}48 \\
40 \\
85\end{array}$ & & & $\begin{array}{l}48 \\
77 \\
75\end{array}$ & $\begin{array}{l}48 \\
77 \\
65\end{array}$ & $\begin{array}{l}0 \\
0\end{array}$ & $\begin{array}{l}\mathbf{0} \\
0 \\
0\end{array}$ & $\frac{7}{-}$ & & $\begin{array}{r}8 \\
6 \\
10\end{array}$ & & $\begin{array}{l}5 \\
9 \\
9\end{array}$ & & $\begin{array}{l}145 \\
135 \\
151\end{array}$ & & & 56 & $1 \cdot 1$ \\
\hline $\begin{array}{c}14 \\
22 / 57\end{array}$ & Pre & $\begin{array}{l}\text { Rest } \\
200 \\
400 \\
\text { Rest } \\
700\end{array}$ & $\begin{array}{r}84 \\
123 \\
165 \\
71 \\
142\end{array}$ & \begin{tabular}{|r|}
332 \\
764 \\
1096 \\
311 \\
1727
\end{tabular} & $\begin{array}{l}8 \\
5 \\
4 \\
9 \\
9\end{array}$ & $\begin{array}{l}50 \\
72 \\
64 \\
49 \\
-\end{array}$ & \begin{tabular}{|r|}
86 \\
169 \\
218 \\
35 \\
117
\end{tabular} & $\begin{array}{r}6.7 \\
10.6 \\
17 \cdot 2 \\
6.4 \\
-\end{array}$ & $\begin{array}{r}3.9 \\
4.5 \\
5.0 \\
8.8 \\
14.7\end{array}$ & $\begin{array}{r}80 \\
86\end{array}$ & $\begin{array}{r}46 \\
37 \\
30 \\
124 \\
103\end{array}$ & $\begin{array}{r}42 \\
58 \\
71 \\
0 \\
\end{array}$ & $\begin{array}{r}0 \\
0 \\
0 \\
27 \\
-\end{array}$ & $\begin{array}{l}\bar{Z} \\
\overline{10} \\
13\end{array}$ & $\frac{146}{\frac{1}{35}}$ & $\frac{12}{5}$ & & $\begin{array}{r}4 \\
3 \\
0 \\
4 \\
12\end{array}$ & $\begin{array}{r}7 \\
7 \\
13\end{array}$ & $\begin{array}{l}145 \\
157 \\
187 \\
142 \\
181\end{array}$ & & - & 62 & $0 \cdot 15$ \\
\hline $01 / 63$ & $\begin{array}{c}\text { Pre } \\
30\end{array}$ & $\begin{array}{l}\text { Rest } \\
200 \\
\text { Rest } \\
400\end{array}$ & $\begin{array}{r}86 \\
151 \\
65 \\
136\end{array}$ & $\begin{array}{r}228 \\
916 \\
245 \\
1245\end{array}$ & $\begin{array}{l}94 \\
86 \\
94 \\
92\end{array}$ & $\begin{array}{r}42 \\
68 \\
43 \\
-\end{array}$ & $\begin{array}{l}38 \\
93 \\
38 \\
83\end{array}$ & $\begin{array}{r}5 \cdot 4 \\
13 \cdot 5 \\
5 \cdot 7 \\
-\end{array}$ & $\begin{array}{r}6.0 \\
9.9 \\
6.5 \\
15.0\end{array}$ & $\begin{array}{l}63 \\
90 \\
88 \\
-\end{array}$ & $\begin{array}{r}70 \\
65 \\
99 \\
111\end{array}$ & $\begin{array}{l}20 \\
+ \\
18 \\
+\end{array}$ & $\frac{28}{26}$ & $\frac{5}{5}$ & $\begin{array}{r}126 \\
178 \\
20 \\
44\end{array}$ & $\begin{array}{r}10 \\
17 \\
6 \\
4\end{array}$ & & $\begin{array}{r}6 \\
9 \\
2 \\
11\end{array}$ & $\begin{array}{r}\overline{9} \\
21\end{array}$ & $\begin{array}{l}114 \\
163 \\
112 \\
159\end{array}$ & 9 & $\begin{array}{r}9 \\
11 \\
8\end{array}$ & 45 & 0.35 \\
\hline $36 / 63$ & $\begin{array}{r}\text { Pre } \\
15\end{array}$ & $\begin{array}{l}\text { Rest } \\
300 \\
\text { Rest } \\
350\end{array}$ & $\begin{array}{r}60 \\
138 \\
65 \\
137\end{array}$ & $\begin{array}{l}223 \\
946 \\
226 \\
941\end{array}$ & $\begin{array}{l}95 \\
80 \\
96 \\
89\end{array}$ & $\begin{array}{l}57 \\
97 \\
51 \\
98\end{array}$ & \begin{tabular}{|r|}
57 \\
131 \\
51 \\
114
\end{tabular} & $\begin{array}{l}3.9 \\
9.8 \\
4.4 \\
9.6\end{array}$ & $\begin{array}{l}3 \cdot 9 \\
7 \cdot 2 \\
4 \cdot 4 \\
8 \cdot 2\end{array}$ & $\begin{array}{l}65 \\
71 \\
68 \\
70\end{array}$ & $\begin{array}{l}65 \\
52 \\
68 \\
60\end{array}$ & $\begin{array}{r}0 \\
27 \\
0 \\
15\end{array}$ & $\begin{array}{l}0 \\
0 \\
0 \\
0\end{array}$ & $\frac{7}{5}$ & $\begin{array}{r}158 \\
228 \\
55 \\
94\end{array}$ & \begin{tabular}{|r|}
12 \\
21 \\
8 \\
16
\end{tabular} & $\overline{\overline{19}}$ & $\overline{71}$ & $\begin{array}{l}10 \\
14\end{array}$ & $\begin{array}{l}130 \\
168 \\
125 \\
133\end{array}$ & $\begin{array}{l}82 \\
73 \\
69\end{array}$ & \begin{tabular}{|r|}
97 \\
114 \\
84 \\
93
\end{tabular} & 45 & 0.5 \\
\hline $\begin{array}{c}17 \\
28 / 53\end{array}$ & Pre & $\begin{array}{c}\text { Rest } \\
\text { Work } \\
\text { Rest } \\
600\end{array}$ & $\begin{array}{r}95 \\
150 \\
92 \\
168\end{array}$ & $\begin{array}{r}158 \\
776 \\
303 \\
1499\end{array}$ & $\begin{array}{l}90 \\
87 \\
98 \\
98\end{array}$ & $\begin{array}{r}49 \\
82 \\
39 \\
104\end{array}$ & \begin{tabular}{|r|}
68 \\
107 \\
39 \\
104
\end{tabular} & $\begin{array}{r}3.2 \\
9.5 \\
7.7 \\
14.4\end{array}$ & $\begin{array}{r}2.3 \\
7.4 \\
7.7 \\
14.4\end{array}$ & $\begin{array}{l}34 \\
6 \\
8 \\
86\end{array}$ & $\begin{array}{l}24 \\
49 \\
84 \\
86\end{array}$ & $\begin{array}{r}22 \\
0 \\
0\end{array}$ & $\begin{array}{l}0 \\
0 \\
0 \\
0\end{array}$ & 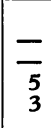 & $\begin{array}{r}125 \\
206 \\
68 \\
141\end{array}$ & $\begin{array}{l}- \\
11 \\
10\end{array}$ & 19 & $\begin{array}{l}9 \\
1 \\
3\end{array}$ & $\begin{array}{r}15 \\
0 \\
0\end{array}$ & $\begin{array}{l}- \\
130 \\
168\end{array}$ & $\begin{array}{l}- \\
90 \\
95\end{array}$ & $\begin{array}{l}- \\
110 \\
130\end{array}$ & 63 & $0 \cdot 16$ \\
\hline
\end{tabular}

value being $16 \mathrm{~mm} . \mathrm{Hg}$ ). Of 8 patients studied in this respect before operation, it was $14 \mathrm{~mm}$. $\mathrm{Hg}$ or more in all but one patient (highest value being 33 $\mathrm{mm} . \mathrm{Hg}$ ). The duration of right ventricular systole, measured from the beginning of isometric contraction to the end of relaxation and expressed as a proportion of the cardiac cycle, decreased after operation in both groups (Table III).

The average resting right ventricular stroke volume increased by $8.5 \mathrm{ml}$. in Group A and $61.6 \mathrm{ml}$. in Group B following operation (Table III). Only the latter difference was significant. In accord with this observation, the arteriovenous oxygen difference in the pulmonary circulation did not change after operation in Group A but was much reduced in Group B (from $61 \mathrm{ml} . / 1$. to $37 \mathrm{ml} . / 1$.). On the other hand, the resting arteriovenous oxygen difference in the systemic circulation did not alter significantly after operation in either group, which means that the systemic output was unchanged.

During exercise, the right ventricular stroke volume in Group B was $54 \mathrm{ml}$. before and $104 \mathrm{ml}$. after operation. Cardiac output during exercise was determined before and after operation in 6 patients of 


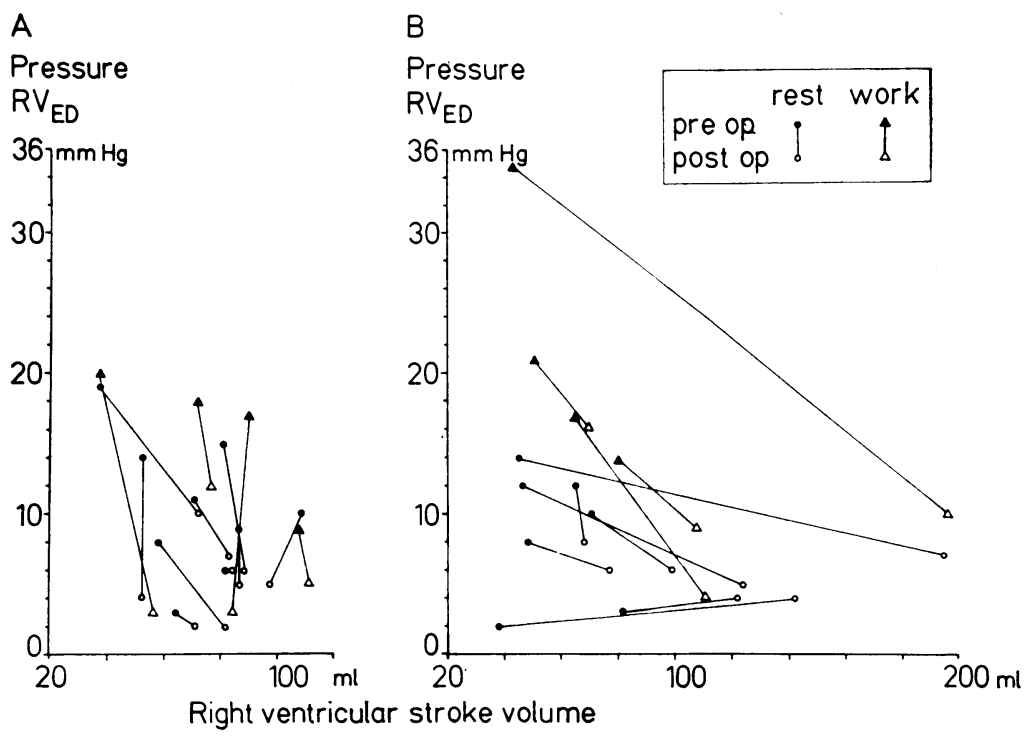

FIG. 1.-End-diastolic pressure in the right ventricle in relation to the stroke volume before and after operation in patients (A) without and (B) with an intra-atrial communication.

Group A. In 3 patients of Group B systemic flow during exercise could not be calculated because of a bidirectional shunt at rest. In most cases the cardiac output was low in relation to the oxygen uptake before as well as after operation (Fig. 2).

An interatrial shunt before operation was found in 7 of the 8 patients belonging to Group B. In 5 (Cases 10,12, 14, 15, and 17) a right-to-left shunt was present at rest, on average amounting to $34 \mathrm{per}$ cent of the systemic flow. In one of these (Case 15) a left-to-right shunt was also present. In 2 patients (Cases 11 and 16) a right-to-left shunt was demonstrated only during exercise. One (Case 13) was not exercised during his pre-operative investigation; a small right-to-left shunt (13\%) was detected during exercise after operation.

Following operation a right-to-left shunt at rest disappeared in 4 out of 5 patients in whom such a

TABLE III

HRMODYNAMIC DATA BEFORE AND AT LATEST POST-OPERATIVE EXAMINATION

\begin{tabular}{|c|c|c|c|c|c|c|}
\hline & $\underset{\left(\mathbf{k p m} / \mathrm{min}^{2}\right)}{\mathrm{PWC}}$ & $\begin{array}{l}\text { Heart vol. } \\
\text { (ml.) }\end{array}$ & $\begin{array}{l}\text { Stroke vol. } \\
\text { (ml.) }\end{array}$ & $\underset{(\mathrm{ml} . / 1 .)}{\mathrm{AV}-\mathrm{O}_{2} \text { diff. }}$ & $\begin{array}{l}\text { Rt. vent. } \\
\text { end-diast. } \\
\text { (mm. Hg) }\end{array}$ & $\begin{array}{l}\text { Rt. vent. } \\
\text { syst. duration } \\
\text { (\% cardiac } \\
\text { cycle) }\end{array}$ \\
\hline $\begin{array}{l}\text { Group } A \\
\text { Pre-operative } \\
\text { SD } \\
\text { Post-operative } \\
\text { SD } \\
\text { Difference, pre-- and post-operative } \\
\text { SD of difference } \\
\text { No. of cases } \\
\text { Probability }\end{array}$ & $\begin{array}{c}609 \\
171 \\
668 \\
176 \\
-58 \\
86 \\
99 \\
>0.05\end{array}$ & $\begin{array}{c}986 \\
296 \\
911 \\
153 \\
75 \\
312 \\
9 \\
>0.4\end{array}$ & $\begin{array}{r}71 \cdot 0 \\
21.3 \\
79.5 \\
13.1 \\
-8.5 \\
137.9 \\
9 \\
>0.8\end{array}$ & $\begin{array}{c}55.8 \\
15.4 \\
48.8 \\
8.1 \\
7.0 \\
15.6 \\
99 \\
>0.2\end{array}$ & $\begin{array}{r}10.5 \\
4.9 \\
5.2 \\
2.5 \\
5.3 \\
3.5 \\
9 \\
<0.01 \\
>0.001\end{array}$ & $\begin{array}{c}52.1 \\
3.2 \\
45.2 \\
4.3 \\
6.9 \\
3.8 \\
9 \\
<0.001\end{array}$ \\
\hline $\begin{array}{l}\text { Group B } \\
\text { Pre-operative } \\
\text { SD } \\
\text { Post-operative } \\
\text { SD } \\
\text { Difference, pre- and post-operative } \\
\text { SD of difference } \\
\text { No. of cases } \\
\text { Probability }\end{array}$ & $\begin{array}{r}469 \\
113 \\
700 \\
305 \\
-231 \\
283 \\
8 \\
>0.05\end{array}$ & $\begin{array}{r}849 \\
371 \\
865 \\
216 \\
-16 \\
222 \\
8 \\
>0.8\end{array}$ & $\begin{array}{r}52 \cdot 3 \\
18 \cdot 8 \\
113.9 \\
41 \cdot 6 \\
-61.6 \\
47.7 \\
8 \\
<0.01 \\
>0.001\end{array}$ & $\begin{array}{r}48 \cdot 0 \\
13.5 \\
41.5 \\
6.6 \\
6.5 \\
10.3 \\
8 \\
>0.1\end{array}$ & $\begin{aligned} 8.7 \\
4.6 \\
5.7 \\
1.5 \\
3.0 \\
3.6 \\
77.05 \\
>0.05\end{aligned}$ & $\begin{array}{r}59.4 \\
8.8 \\
48.5 \\
3.9 \\
10.9 \\
7.0 \\
8 \\
<0.01 \\
>0.001\end{array}$ \\
\hline
\end{tabular}


shunt had been present. In 1 patient who had a bidirectional shunt before operation (Case 15) a small right-to-left shunt $(18 \%)$ remained. A leftto-right shunt (mean 28\%) was demonstrated in 4 patients. Three of these patients (Cases 10, 12 , and 14) showed no evidence of this shunt before operation. Comparison of the right-to-left shunt before and after operation, during exercise, was not possible since systemic flow during exercise could not be calculated in patients with a left-toright shunt at rest.

In Group B the mean arterial oxygen saturation at rest was 91 per cent before and 97 per cent after operation. During exercise, the mean arterial oxygen saturation was 77 per cent before and 93 per cent after operation, indicating that the right-toleft shunt during exercise was much reduced after operation. This occurred in spite of the exercise being somewhat more severe during the postoperative investigation (Table II).

The mean physical working capacity was $609 \mathrm{kpm} /$ min. before and $668 \mathrm{kpm} / \mathrm{min}$. after operation in Group $A$ and the corresponding values for Group $B$ were 469 and $700 \mathrm{kpm} / \mathrm{min}$. These differences were not statistically significant (Table III). The larger difference in Group B results mainly from a large rise in one patient (Case 9) and a moderate rise in two others (Cases 10 and 14). The average heart volume did not change significantly after operation (Table III). However, in only 2 patients were the hearts much enlarged before operation. Case 3, with the highest resting right ventricular systolic and end-diastolic pressures, had a heart volume of $1600 \mathrm{ml}$. before operation and a normal volume of $760 \mathrm{ml}$. three years after operation. In another patient (Case 12), who also had a markedly increased right ventricular systolic pressure, the heart volume decreased from 1570 to $1070 \mathrm{ml}$. three years after operation. This figure was normal in relation to his post-operative physical working capacity.

\section{Discussion}

The oxygen transport capacity (aerobic working capacity) is a function of the stroke volume (SV), arteriovenous oxygen difference (AVD), and heart rate (f), as expressed in Fick's equation: $\mathrm{Vo}_{2}=$ $f \times S V \times A V D$. Therefore, for a given heart rate during exercise the working capacity depends on the stroke volume and the arteriovenous oxygen difference, i.e. oxygen pulse. In patients with severe valvular heart disease with a resultant small stroke volume, such as found in tight mitral, pulmonary, or aortic stenosis (Granath, 1965; Ikkos et al., 1966; Lee et al., 1967), a large arteriovenous oxygen difference is found. This observation has

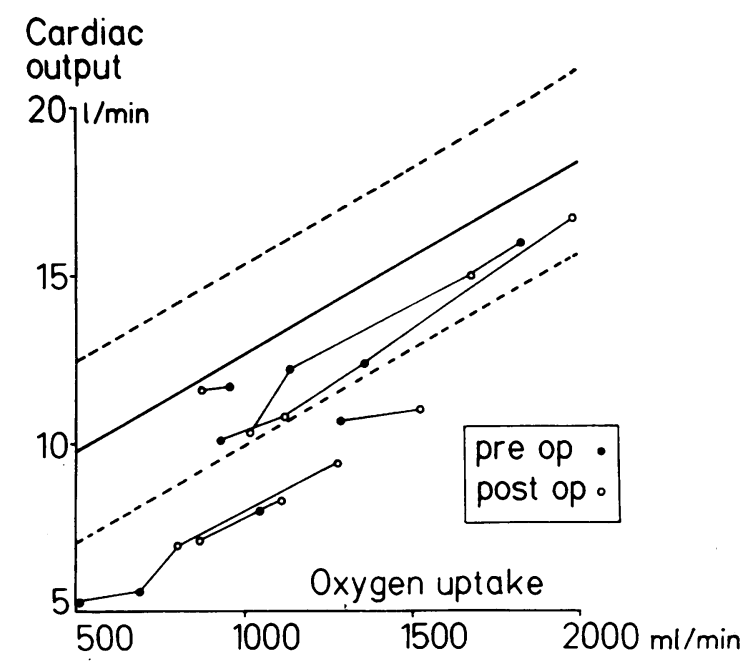

FIG. 2.-Cardiac output in relation to oxygen uptake during exercise before and after operation in 6 patients in Group A. The continuous and broken lines indicate the regression line \pm and two standard errors of estimate, respectively, in normal subjects (calculated from data published by Bevegård, Holmgren, and Jonsson, 1960; Holmgren, Jonsson, and Sjöstrand, 1960).

also been made in complete atrioventricular block with normal or increased stroke volume but low cardiac output (Bevegård et al., 1967). Thus a high arteriovenous oxygen difference is an adaptation in order to maintain an oxygen transport in situations where cardiac output is reduced. A similar adaptation is also found during intensive physical training (Åstrand et al., 1965; Andrew, Guzman, and Becklake, 1966; Holmgren et al., 1957).

Because of these phenomena, the physical working capacity was normal in spite of the low stroke volume during exercise in most patients in Group A before operation, and showed no increase after operation. However, in patients in Group B in whom a right-to-left shunt was present at rest as well as during exercise, resulting in reduced arterial oxygen content, a low working capacity was present before operation and an increase was found after operation. This was mainly due to the change in arterial oxygen saturation during exercise from 77 before to 93 per cent after operation.

This study has confirmed the persistence of a low cardiac output during exercise following pulmonary valvotomy, as reported by Johnson (1962). It has been suggested (Johnson, 1962; McIntosh and Cohen, 1963) that the low cardiac output after pulmonary valvotomy is due to a persistent myocardial fibrosis and a consequent resistance to right ventricular filling. This was not, however, the finding 
in the present study because the right ventricular end-diastolic pressure decreased considerably after operation. Further evidence against this arises from our observation that right-to-left interatrial shunts before operation changed in direction and became left-to-right shunts after operation, with an increase in right ventricular stroke volume and the right ventricular systolic duration shortened.

If one accepts the theory that cardiac output is primarily regulated to supply the tissue oxygen requirement (Guyton, 1963), the increased peripheral oxygen extraction may be interpreted as an adaptational phenomenon in patients unable to maintain a normal cardiac output and the adaptation persists after operation. This is in agreement with the observation made in some patients with tight mitral stenosis who did not show any increase in cardiac output following successful mitral commissurotomy (Granath, 1965; Donald et al., 1957).

The limiting factor for restoring a normal cardiac output after operation may be the size of the left ventricle which is expected to be small as the pulmonary stenosis has been present since birth. From this study it is evident that in cases with an intra-atrial communication the right ventricle is able to increase its stroke volume after the stenosis is relieved. However, the left ventricle must also increase its stroke volume and this may be possible only by intensive physical training, giving rise to periods with a high left ventricular filling pressure.

\section{SUMMARY}

Seventeen patients with pulmonary stenosis and an intact ventricular septum were studied before and after operation. Nine patients had an intact atrial septum and 8 had an interatrial shunt. The physical working capacity and the haemodynamic effects of exercise were examined. A low cardiac output during exercise in relation to the oxygen uptake persisted after operation. This did not appear to be the result of irreversible damage of the right ventricular myocardium because the ventricular end-diastolic pressure decreased considerably, the duration of systole shortened, and a rise in right ventricular stroke volume at rest and during exercise was demonstrated in those patients with interatrial shunts.

It was concluded that the tendency for low cardiac output during exercise after operation was a result of an adaptation of peripheral circulation, from early childhood, resulting in the ability to extract and utilize oxygen to a greater degree. Once such an adaptation has occurred there may be no necessity to increase cardiac output to meet the demand for oxygen transport. The importance of a persisting small left ventricle is discussed.
An appreciable increase of the physical working capacity was shown only in those patients who had a right-to-left shunt before operation. This was attributed to the increased arterial oxygen saturation during exercise, as a result of the diminished rightto-left shunt at the atrial level after operation. The considerable reduction in the right ventricular enddiastolic pressure was apparently responsible for the decreased right-to-left shunt. In patients without an interatrial shunt, physical working capacity was generally normal before operation and did not change after operation. The normal working capacity in the presence of reduced stroke volume during exercise was possible because of the widened arteriovenous oxygen difference.

\section{REFERENCES}

Andrew, G. M., Guzman, C. A., and Becklake, M. R. (1966). Effect of athletic training on exercise cardiac output. F. appl. Physiol., 21, 603.

Åstrand, P. O., Ekblom, D., Messin, R., Saltin, B., Stenberg, J., and Wallström, N. B. (1965). Effect of training on circulation during exercise. 23rd International Congress of Physiol. Science, Tokyo, p. 308.

Bevegård, S., Holmgren, A., and Jonsson, B. (1960). The effect of body position on the circulation at rest and during exercise, with special reference to the influence on the stroke volume. Acta physiol. scand., 49, 279.

-, Jonsson, B., Karlöf, I., Lagergren, H., and Sowton, E. (1967). Effect of changes in ventricular rate on cardiac output and central pressures at rest and during exercise in patients with artificial pacemakers. Cardiovasc. Res., $1,21$.

Donald, K. W., Bishop, J. M., Wade, O. L., and Wormald, P. N. (1957). Cardiorespiration function two years after mitral valvotomy. Clin. Sci., 16, 325.

Granath, A. (1965). Mitral valvulotomy. A clinical and hemodynamic pre- and postoperative study. Acta med. scand., 178, Suppl. 433.

Guyton, A. C. (1963). Circulatory Physiology: Cardiac Output and its Regulation, p. 301. Saunders, Philadelphia and London.

Holmgren, A., Jonsson, B., Levander, M., Linderholm, H., Mossfeldt, F., Sjöstrand, T., and Ström, G. (1957). Physical training of patients with vasoregulatory asthenia. Acta med. scand., 158, 437.

$\longrightarrow,-$ and Sjöstrand, T. (1960). Circulatory data in normal subjects at rest and during exercise in recumbent position, with special reference to the stroke volume at different work intensities. Acta physiol. scand., 49, 343.

Ikkos, D., Jonsson, B., and Linderholm, H. (1966). Effect of exercise in pulmonary stenosis with intact ventricular septum. Brit. Heart f., 28, 316.

Johnson, A. M. (1962). Impaired exercise response and other residua of pulmonary stenosis after valvotomy. Brit. Heart F., 24, 375.

Lee, S., Bevegård, S., Jonsson, B., Karlöf, I., and Åström, H. (1967). Physical working capacity and hemodynamic effects of exercise in aortic stenosis. Canad. med. Ass. $\boldsymbol{F}$. In the press.

McIntosh, H. D., and Cohen, A. I. (1963). Pulmonary stenosis: The importance of the myocardial factor in determining the clinical course and surgical results. Amer. Heart F., 65, 715. 